\title{
Evidence suggests that water fluoridation has an effect over and above that of fluoridated toothpaste
}

\author{
McDonagh M, Whiting P, Bradley M, Cooper J, Sutton A, Chestnutt I, et al. A Systematic Review of Public Water \\ Fluoridation. York: Publications Office, NHS Centre for Reviews and Dissemination, University of York. \\ ISBN 190064016 3; 2000
}

Objective If water fluoridation is shown to have beneficial effects, what is the effect over and above that offered by the use of alternative interventions and strategies?

\section{Data sources See page 37.}

Study selection Studies were included that were carried out after 1974 in industrialised countries because, in those, any modifying effect of fluoride toothpaste and other sources of dental fluoride (eg, mouthrinses, tablets) should be apparent. Of the 24 studies that met the inclusion criteria for Objective 1, 10 were completed after 1974 (1978-1997). The mean validity score of these 10 studies was 5.0 (range, 3.5-6.8 out of 8).

Data extraction and synthesis See page 37.

Results Only 10 studies qualified to address this issue, although none of these identified this objective as the purpose of the investigation. The study with the highest validity score showed a statistically-significant difference in caries increment between fluoridated and nonfluoridated areas. Those in the nonfluoridated area had the greatest increment, in spite of fluoridated toothpaste being used by both groups ( $94 \%$ versus $95 \%$ used fluoride toothpaste only in the fluoridated and nonfluoridated groups, respectively).

Conclusions The evidence suggests that water fluoridation has an effect over and above that of fluoridated toothpaste (and other sources of fluoride).

Evidence-Based Dentistry (2002) 3, 40. doi:10.1038/sj. ebd. 6400118

Address for reprints: Publications Office, NHS Centre for Reviews and Dissemination, University of York, York YO10 5DD, UK. Tel: +44 (0)1904 433648; fax: +44 (0)1904 433661; e-mail: crdpub@york.ac.uk

\section{Commentary}

A range of other sources of fluoride are available and have been used by dentists in order to reduce caries levels. These include fluoridated milk, salt and fruit juice, as well as mouthwashes, varnishes, tablets, drops and toothpastes. This section of the York review focuses on the most commonly available source of fluoride, toothpastes.

The studies included in this section are a subset of those included to answer the question of whether fluoride reduces caries prevalence. The reviewers used a cut-off point of 1974 because after this time more than $50 \%$ of the toothpastes on the market in the UK contained fluoride, with fluoride toothpastes gaining $96 \%$ of the market by $1976 .{ }^{1}$

None of the studies included were specifically designed to determine if water fluoridation had any beneficial effects over and above alternative strategies. Only one of the studies, that by
Hardwick et al. ${ }^{2}$ which had the highest validity score for any of the investigations included, reported the use of fluoridated toothpaste in both groups. People in the nonfluoridated area showed the biggest caries increment in spite of fluoridated toothpaste being used by both fluoridated and nonfluoridated groups (94\% and $95 \%$, respectively).

It has been suggested that total fluoride exposure has increased over recent years, particularly in industrialised nations. Exposure to fluoride from sources other than water may alter the amount required in water for optimum caries reduction and is thus a potential confounding factor in studies of the association between water fluoridation and negative effects. Some studies ${ }^{3,4}$ have measured total fluoride exposure in people exposed to fluoridated and nonfluoridated water. Exposure to sources of fluoride varies, however, requiring measurement at an individual level not a population one.
The small number and moderate quality of included studies for this section of the review means that we can be no more certain than saying that the evidence suggests that water fluoridation has an effect over and above that of fluoridated toothpaste.

1. Murray JJ, Rugg-Gunn AJ, Jenkins GN (eds). Fluorides in Caries Prevention. 3rd Edn. Oxford: Butterworth-Heinemann; 1991.

2. Hardwick J, Teasdale J, Bloodworth G. Caries increments over 4 years in children aged 12 at the start of water fluoridation. Br Dent J 1982; 153:217-222

3. Guha-Chowdhury N, Drummond B, Smillie AC. Total fluoride intake in children aged 3 to 4 years - a longitudinal study. J Dent Res 1996; 75:1451-1457.

4. Mansfield $P$. The distribution of urinary fluoride concentration in the UK. Fluoride 1999; 32:27-32.

Jeyanthi H John Centre for Evidence-based Dentistry, Institute of Health Sciences, Oxford, UK 